\title{
The Radio Pulsar J0205+6449 in the SNR 3C 58
}

\author{
V. M. Malofeev, I. F. Malov, O. I. Malov, A. P. Glushak \\ Lebedev Physical Institute, Moscow, Russia
}

\begin{abstract}
Pulsed radio emission has been detected from the X-ray pulsar J0205+6449 in the SNR 3C 58 at 111 and $88 \mathrm{MHz}$ on radio telescopes of the Pushchino Radio Astronomy Observatory. The dispersion measure $\mathrm{DM}=141 \mathrm{pc} \mathrm{cm}^{-3}$ has been confirmed. The synchrotron mechanism is proposed for the radio and X-ray emission to explain the lower X-ray and radio luminosities of this pulsar compared to the Crab pulsar.
\end{abstract}

\section{Results}

Murray et al. (2002) reported the detection of an X-ray pulsar J0205+6449 with a period of $65.68 \mathrm{~ms}$ in the SNR 3C 58. Estimates of the distance to the SNR range from $1.5 \mathrm{kpc}$ (Losinskaya 1986) to more than $6.5 \mathrm{kpc}$ (Williams 1973).

Using the pulsar search program and the sensitive Pushchino Large Phased Array we detected pulsed signals from PSR J0205+6449 at $111 \mathrm{MHz}$ (Malofeev et al. 2001). Later similar signals were registered by the other Pushchino radio telescope DKR-1000 at $88 \mathrm{MHz}$. Camilo et al. (2002) detected PSR J0205+6449 at 820 and $1375 \mathrm{MHz}$ and measured its dispersion measure $\mathrm{DM}=140.7 \pm 0.3 \mathrm{pc}$ $\mathrm{cm}^{-3}$. Our data confirmed that the best profile can be obtained if DM $=141 \pm 10$ pc $\mathrm{cm}^{-3}$ (Malofeev et al. 2003 and Fig. 1). This value of DM gives the distance to the pulsar of $6.4 \mathrm{kpc}$. Figure 2 shows the integrated profile of PSR J0205+6449 at $111.23 \mathrm{MHz}$. The measured parameters of this pulsar are listed in Table 1. Using a longer MJD interval (52164-52779) we obtained more precise values of $P$ and $\dot{P}$ than Camilo et al. (2002) and Malofeev et al. (2003).

The synchrotron model is proposed to explain the difference between the luminosities of PSR J0205+6449 and the Crab pulsar. In this model the radio luminosity $L_{R}$ is proportional to $B_{L C}$ (Malov 1999) and since this radio emission is coherent, $L_{R} \propto n^{2} B_{L C}$. We can use the Goldreich-Julian formula for the density of relativistic particles:

$$
n=\frac{B}{P c e}=\frac{8 \pi^{3} R_{*}^{3} B_{s}}{c^{4} e P^{4}}
$$

The period of PSR J0205+6449 is two times more than the Crab pulsar's period. This means that the radio emission of PSR J0205+6449 should be a factor of $\sim 2000$ weaker than from PSR B0531+21, in agreement with observations. The $\mathrm{X}$-ray radiation is not coherent and $L_{x} \propto n B^{(\alpha+1) / 2}$ (Pacholczyk 1970). For the spectral index $\alpha=2.8$ the ratio of the X-ray luminosities of the Crab pulsar and of PSR J0205+6449 should be $\sim 1000$, also in good agreement with observations.

The correlation between the optical bolometric luminosities and the values of $B_{L C}$ and the similar correlation for the hard radiation (X-rays \& $\gamma$-rays), 
namely $\log L_{\text {opt }}=(1.85 \pm 0.47) \log B_{L C}+(21.00 \pm 2.32)$ and $\log L_{x+\gamma}=(0.83 \pm$ $0.14) \log B_{L C}+(31.20 \pm 0.70)$, enable us to predict for PSR J0205+6449 $L_{\text {opt }} \sim$ $10^{31} \mathrm{ergs} \mathrm{s}^{-1}$ and $L_{x+\gamma}=7 \times 10^{35} \mathrm{ergs} \mathrm{s}^{-1}$, making searches for optical emission and gamma-rays from this pulsar promising.

Acknowledgments. This work was partly supported by RFBR (PrÑo. 0302-16509), INTAS (Grant No. 00-00849), an IAU Travel Grant and by the NSF (Grant No. 00-098685).

Table 1. The measured parameters of PSR J0205+6449.

\begin{tabular}{ccccc}
\hline $\mathrm{P}(\mathrm{ms})$ & $\dot{P}$ & $\mathrm{MJD}$ & \multicolumn{2}{c}{$\left.\mathrm{DM}(\mathrm{pc} \mathrm{cm})^{-3}\right)$} \\
\hline $65.67895013(6)$ & $1.93498(1) \times 10^{-13}$ & 51901.330 & \multicolumn{2}{c}{$141 \pm 10$} \\
\hline & & & & \\
\hline$S_{111}(\mathrm{mJy})$ & $S_{88}(\mathrm{mJy})$ & $w_{50}(111 \mathrm{MHz})(\mathrm{ms})$ & $w_{10}(111 \mathrm{MHz})(\mathrm{ms})$ & $\alpha$ \\
\hline 40 & $<150$ & $\sim 25$ & $\sim 40$ & 2.8 \\
\hline
\end{tabular}

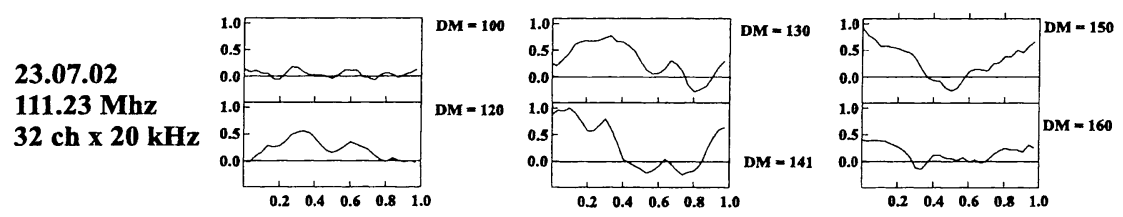

Figure 1. Sample of integrated pulsar profiles of PSR J0205+6449 for various dispersion measures.
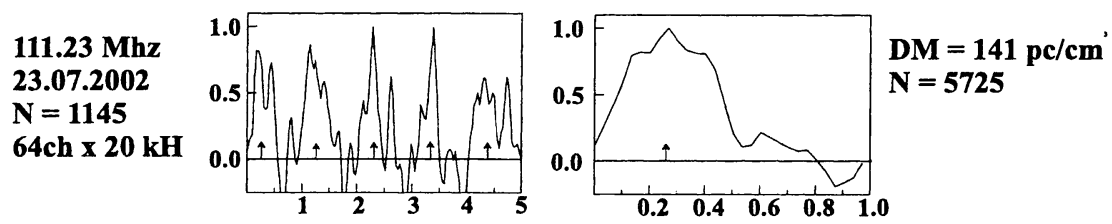

Figure 2. The integrated profiles of PSR J0205+6449: the observing window $\left(P_{0}\right)$ equals five apparent periods $(P=65.68 \mathrm{~ms})$, this profile is obtained by the integration over $1145 P_{0}$ (left); integrated profile obtained by the folding with 5725 apparent periods (right).

\section{References}

Camilo, F. et al. 2002, ApJ, 571, L41

Losinskaya, T. A. 1986, Supernovae and Stellar Winds, (Moscow: Nauka) Malofeev, V. M., Malov, O. I., \& Glushak, A. P. 2001, IAU Circ. No. 7775 Malofeev, V. M. et al. 2003, Astron. Reports, 47, 413

Malov, I. F. 1999, Astron. Reports, 43, 727

Murray, S. et al. 2002, ApJ, 568, 226

Pacholczyk, A. G. 1970, Radio Astrophysics, (San Francisco: Freeman)

Williams, D. R. W. 1973, A\&A, 28, 309 